\title{
Co-crystallization with Dab antibody fragments: A universal method for the introduction of synthetic symmetry
}

\author{
Chelsy Chesterman ${ }^{1}$, Eddy Arnold ${ }^{2}$ \\ ${ }^{1}$ GSK Vaccines, 14200 Shady Grove Road, Rockville MD 20850, chelsy.chesterman@outlook.com \\ ${ }^{2}$ Rutgers, The State University of New Jersey, 679 Hoes Lane West, Piscataway NJ 08854, arnold@rutgers.cabm.com
}

The introduction of synthetic symmetry by design is a potentially valuable strategy for the crystallization of challenging targets. Previous studies have explored multiple strategies to create an artificial protein dimer, but they all require introducing specific mutations on the target protein's surface (1-3). This work demonstrates a new method for achieving symmetry through co-crystallization with an antibody fragment termed a diabody (Dab). These Dabs contain two complete target binding sites and can be used to form a symmetrical complex with two identical bound proteins (4-6). By avoiding the need for specific surface mutations on a target protein, Dabs present a general platform that requires no knowledge of target protein structure. In addition, antibody fragments have a long history of success as crystallization chaperones. Therefore, this strategy is broadly applicable to a wide range of difficult targets, especially when monoclonal antibodies for a target protein have previously been identified.

A library of Dabs has been generated, which includes flexible Dabs and Dabs containing specific disulfide bonds at the interface of the antibody dimer. The introduction of a disulfide bond promotes a preferred conformation in solution and an increased likelihood of crystallization. Three Dabs with disulfide bonds and a flexible control Dab have been characterized both in solution and through high-resolution crystal structures (1.4 $-1.6 \AA$ ). To explore the capacity of engineered Dabs to enable the crystallization of other proteins through synthetic symmetry, we generated complexes between the engineered Dabs and HIV-1 reverse transcriptase (RT) bound to a high-affinity DNA template-primer aptamer. Symmetrical complexes containing Dabs and bound RT-aptamer produced crystals over a wider range of precipitant conditions than RT-aptamer alone. After optimization, crystals of a Dab-RT-aptamer complex were diffraction quality, allowing structure determination at 3.0 Å resolution.

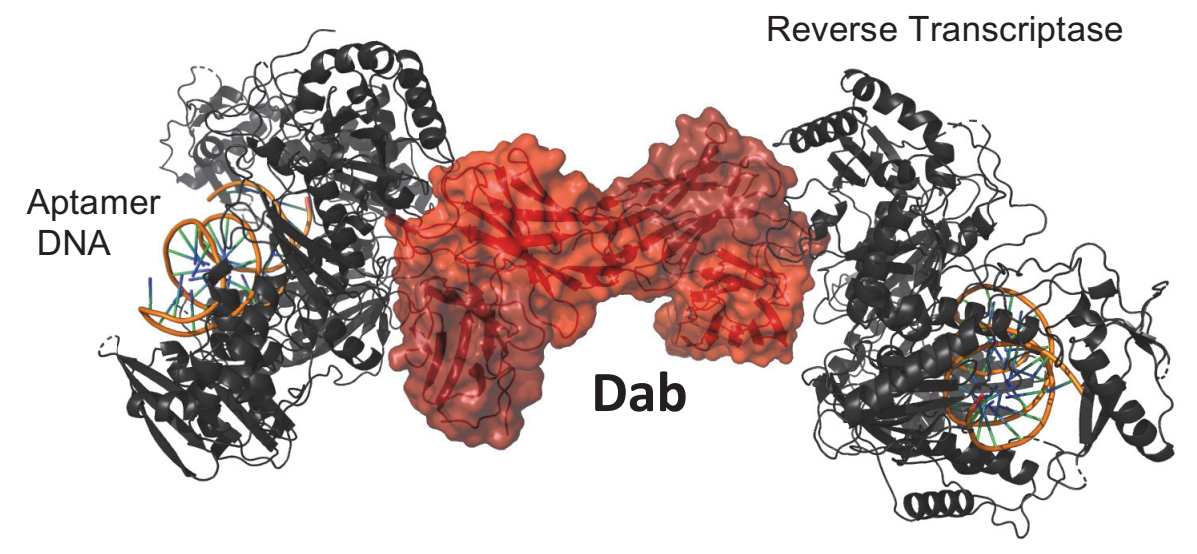

Figure 1:

Crystal structure of a Dab bound to two copies of a HIV-1 RT-aptamer protein complex.

\author{
Acknowledgements \\ as a post-doctoral fellow. \\ References \\ 1. Forse, G.J., et al. (2011) Protein Science, 20, 168-178. \\ 2. Laganowsky, A., et al. (2011) Protein Science, 20, 1876-90. \\ 3. Yamada, H., et al. (2007) Protein Science, 16, 1389-1397. \\ 4. Holliger, P., et al. (1993) PNAS, 90, 6444-6448. \\ 5. Todorovska, A., et al. (2001) Journal of Immunological Methods, 248, 47-66. \\ 6. Kim, J.H., et al. (2016) Scientific Reports, 6, 1-12.
}

This work was funded by NIH MERIT Award AI027690 to E. Arnold and completed at Rutgers University. X-ray diffraction data were collected at the Advanced Photon Source, the Stanford Synchrotron Radiation Source, and the National Synchrotron Light Source II. This work was conducted prior to author Chelsy Chesterman's current engagement with GSK 